\title{
In-medium hadron properties measured with HADES
}

J. Pietraszko ${ }^{3, a}$ and G. Agakishiev ${ }^{6}$, C. Behnke ${ }^{7}$, D. Belver ${ }^{16}$, A. Belyaev ${ }^{6}$, J.C. Berger-Chen ${ }^{8}$, A. Blanco ${ }^{1}$, C. Blume ${ }^{7}$, M. Böhmer ${ }^{9}$, P. Cabanelas ${ }^{16}$, S. Chernenko ${ }^{6}$, C. Dritsa ${ }^{10}$, A. Dybczak ${ }^{2}$, E. Epple ${ }^{8}$, L. Fabbietti ${ }^{8}$, O. Fateev ${ }^{6}$, P. Fonte ${ }^{1, a}$, J. Friese ${ }^{9}$, I. Fröhlich ${ }^{7}$, T. Galatyuk ${ }^{4, b}$, J. A. Garzón ${ }^{16}$, K. Gill', M. Golubeva ${ }^{11}$, D. González-Díaz ${ }^{4}$, F. Guber ${ }^{11}$, M. Gumberidze ${ }^{4}$, S. Harabasz ${ }^{4,2}$, T. Hennino ${ }^{14}$, C. Höhne ${ }^{10}$, R. Holzmann ${ }^{3}$, P. Huck ${ }^{9}$, A. lerusalimov ${ }^{6}$, A. Ivashkin ${ }^{11}$, M. Jurkovic ${ }^{9}$, B. Kämpfer ${ }^{5, c}$, T. Karavicheva ${ }^{11}$, I. Koenig ${ }^{3}$, W. Koenig ${ }^{3}$, B. W. Kolb ${ }^{3}$, G. Korcyl ${ }^{2}$, G. Kornakov ${ }^{4}$, R. Kotte ${ }^{5}$, A. Krása ${ }^{15}$, E. Krebs ${ }^{7}$, F. Krizek ${ }^{15}$, H. Kuc ${ }^{2,14}$, A. Kugler ${ }^{15}$, A. Kurepin ${ }^{11}$, A. Kurilkin ${ }^{6}$, P. Kurilkin ${ }^{6}$, V. Ladygin ${ }^{6}$, R. Lalik ${ }^{8}$, S. Lang ${ }^{3}$, K. Lapidus ${ }^{8}$, A. Lebedev ${ }^{12}$, L. Lopes ${ }^{1}$,

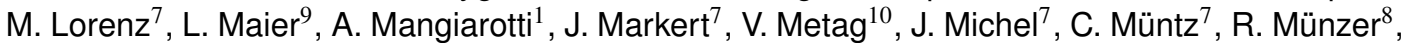
L. Naumann ${ }^{5}$, M. Palka ${ }^{2}$, Y. Parpottas ${ }^{13, d}$, V. Pechenov ${ }^{3}$, O. Pechenova ${ }^{7}$, W. Przygoda ${ }^{2}$, B. Ramstein ${ }^{14}$, L. Rehnisch ${ }^{7}$, A. Reshetin ${ }^{11}$, A. Rustamov ${ }^{7}$, A. Sadovsky ${ }^{11}$, P. Salabura ${ }^{2}$, T. Scheib ${ }^{7}$, H. Schuldes ${ }^{7}$, J. Siebenson ${ }^{8}$, Yu.G. Sobolev ${ }^{15}$, S. Spataro ${ }^{e}$, H. Ströbele ${ }^{7}$, J. Stroth ${ }^{7,3}$, P Strzempek ${ }^{2}$, C. Sturm ${ }^{3}$, O. Svoboda ${ }^{15}$, A. Tarantola ${ }^{7}$, K. Teilab ${ }^{7}$, P. Tlusty ${ }^{15}$, M. Traxler ${ }^{3}$,

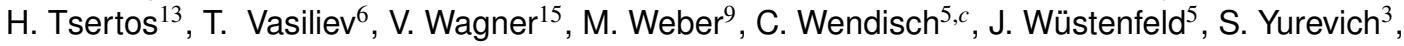
Y. Zanevsky ${ }^{6}$

\section{(HADES collaboration)}

${ }^{1}$ LIP-Laboratório de Instrumentação e Física Experimental de Partículas , 3004-516 Coimbra, Portugal

${ }^{2}$ Smoluchowski Institute of Physics, Jagiellonian University of Cracow, 30-059 Kraków, Poland

${ }^{3}$ GSI Helmholtzzentrum für Schwerionenforschung GmbH, 64291 Darmstadt, Germany

${ }^{4}$ Technische Universität Darmstadt, 64289 Darmstadt, Germany

${ }^{5}$ Institut für Strahlenphysik, Helmholtz-Zentrum Dresden-Rossendorf, 01314 Dresden, Germany

${ }^{6}$ Joint Institute of Nuclear Research, 141980 Dubna, Russia

${ }^{7}$ Institut für Kernphysik, Goethe-Universität, 60438 Frankfurt, Germany

${ }^{8}$ Excellence Cluster 'Origin and Structure of the Universe', 85748 Garching, Germany

${ }^{9}$ Physik Department E12, Technische Universität München, 85748 Garching, Germany

${ }^{10}$ II.Physikalisches Institut, Justus Liebig Universität Giessen, 35392 Giessen, Germany

${ }^{11}$ Institute for Nuclear Research, Russian Academy of Science, 117312 Moscow, Russia

${ }^{12}$ Institute of Theoretical and Experimental Physics, 117218 Moscow, Russia

${ }^{13}$ Department of Physics, University of Cyprus, 1678 Nicosia, Cyprus

${ }^{14}$ Institut de Physique Nucléaire (UMR 8608), CNRS/IN2P3 - Université Paris Sud, F-91406 Orsay Cedex, France

${ }^{15}$ Nuclear Physics Institute, Academy of Sciences of Czech Republic, 25068 Rez, Czech Republic

${ }^{16}$ LabCAF. F. Física, Univ. de Santiago de Compostela, 15706 Santiago de Compostela, Spain

${ }^{a}$ also at ISEC Coimbra, Coimbra, Portugal

${ }^{b}$ also at ExtreMe Matter Institute EMMI, 64291 Darmstadt, Germany

${ }^{c}$ also at Technische Universität Dresden, 01062 Dresden, Germany

${ }^{d}$ also at Frederick University, 1036 Nicosia, Cyprus

${ }^{e}$ also at Dipartimento di Fisica Generale and INFN, Università di Torino, 10125 Torino, Italy

\footnotetext{
ae-mail: j.pietraszko@gsi.de
} 


\begin{abstract}
Many QCD based and phenomenological models predict changes of hadron properties in a strongly interacting environment. The results of these models differ significantly and the experimental determination of hadron properties in nuclear matter is essential. In this paper we present a review of selected physics results obtained at GSI Helmholtzzentrum für Schwerionenforschung GmbH by HADES (High-Acceptance DiElectron Spectrometer). The $e^{+} e^{-}$pair emission measured for proton and heavy-ion induced collisions is reported together with results on strangeness production. The future HADES activities at the planned FAIR facility are also discussed.
\end{abstract}

\title{
1 Introduction
}

Investigating the properties of nuclear matter in a strongly interacting environment is one of the main objectives in relativistic heavy-ion physics. Different theoretical approaches predict that the vacuum properties of a single hadron are modified once it is placed into nuclear matter. To explore these phenomena an appropriate experimental approach, sensitive to the in-medium effects is essential. The low mass vector mesons $\rho, \omega$ are well suited probes for such a study. Their short life time and the decays of vector mesons into di-electrons offer a unique experimental way to learn about the in-medium modifications, as the di-electrons carry out undisturbed information from the dense and hot nuclear matter. On the other hand, the measurement and reconstruction of electromagnetic channels are challenging, mainly due to the huge hadron background and low branching ratios. Additionally, the dileptons measured in the detection system encode information about all emission processes integrated over the whole collision history. With precise knowledge of particle yields at chemical freeze-out, obtained from hadronic observables, it is however possible to subtract the late contributions from the pair spectrum. Additionally, using elementary collisions one can learn about the contribution from first-chance collisions. Combining all of the above the in-medium component can be reconstructed.

Another, very attractive experimental probe to study the high density phase of nuclear matter are strange particles. In particular, from the subthreshold $\mathrm{K}^{+}$production tight constraints can be put on the nuclear equation of state at matter densities of 2-3 times normal nuclear density $\left(\rho_{0}\right)$ [1]. Furthermore, it has been demonstrated that the kaon phase space distributions and flow patterns are sensitive to the in-medium kaon potential [2].

The High Acceptance Di-Electron Spectrometer (HADES) [3] located at the heavy-ion synchrotron SIS18 at GSI Helmholzzentrum für Schwerionenforschung in Darmstadt, Germany, offers a unique capability for performing high quality di-electron and hadron identification with very good performance of vertex reconstruction, which is essential for measuring off-vertex decays. In the design of the spectrometer, particular emphasis has been given to a good mass resolution $\left(\delta M_{i n v} / M_{i n v} \simeq\right.$ $1 \%(\sigma))$, an electron-positron, proton and pion identification capability over a broad momentum range $(0.1<\mathrm{p}<1.5 \mathrm{GeV} / \mathrm{c})$ and a large geometrical acceptance $\left(\Omega_{\text {pair }} \simeq 35 \%\right.$ of full solid angle at $\left.\mathrm{M}_{\text {pair }} 0.5 \mathrm{GeV}\right)$. The device is described in details in reference [3]. This report reviews the recent HADES experimental findings in the dilepton and hadron sector and is organized as follows: In section 2 a description of dilepton pair production in heavy-ion and proton induced reactions is given. Section 3 reports on strangeness production measured with HADES. The future HADES at FAIR (SIS100) experiments are described in section 4. Finally, the paper is summarized in section 5. 


\section{Di-electron probes}

The observation of an enhanced yield of low-mass lepton pairs $\left(\mathrm{M}_{\text {pair }}<1 \mathrm{GeV} / \mathrm{c}^{2}\right)$ extends back over more than two decades. The enhanced yield, defined as the excess above the contribution expected from well known long-lived hadronic sources, was reported by several groups for reactions in a large range of bombarding energies. Starting in the Bevalac energy regime (1-2 A GeV) with the DLS experiment [4] it continued with measurements of the CERES [5] and NA60 [6] collaborations at low and top SPS energies. While the excess observed at SPS energies could fairly well be described by different transport models involving a significant in-medium modification of the rho meson spectral function in the hadronic medium [7], the results obtained at lower beam energies of 1-2 A GeV [4] could not be satisfactorily reproduced by theoretical models which led to the so-called "DLS-puzzle" [8].

The HADES collaboration has therefore started a systematic investigation of dilepton production in the SIS18 energy regime of 1-2 A GeV. In the beginning, the focus was on light collision systems to revisit the "DLS-puzzle". In order to verify the DLS findings, an experiment was conducted in which a $1 \mathrm{~A} \mathrm{GeV}^{12} \mathrm{C}$ beam was incident onto a carbon target [9]. The measured inclusive di-electron production in the invariant pair mass region $0.15 \mathrm{GeV} / \mathrm{c}^{2}<\mathrm{M}_{e e}<0.50 \mathrm{GeV} / \mathrm{c}^{2}$ was considerably stronger than expected from the known contribution of $\eta$ meson decays. A comprehensive comparison of the HADES and DLS results was achieved by a procedure taking the very different acceptances and trigger conditions of both experiments [9] into account. The comparison of differential cross sections for invariant pair masses and transverse momenta confirmed a good agreement of both measurements [9] indicating that indeed the theoretical interpretation of the data based on model descriptions needs a better understanding of the processes important at this beam energy e.g. decays of the $\mathrm{N}^{*}$ resonances, like $\mathrm{N}^{*}(1520)$ and $\mathrm{N}^{*}(1720)$, and their strong coupling to the $\rho$ meson [10].

\subsection{Low- and intermediate-mass region}

The dielectron production in $\mathrm{N}+\mathrm{N}$ collisions in the SIS18 energy range (1-2 A GeV) is mainly determined by the following processes: (1) decays of baryonic resonances, mainly $\Delta(1232)$, (2) N-N bremsstrahlung process and (3) decays of $\pi^{0}$ and $\eta$ mesons [11]. A detailed knowledge about all these processes is essential for the interpretation of data from heavy ion and proton induced reactions in the SIS18 energy range.

The HADES high quality results on dilepton production in $\mathrm{p}+\mathrm{p}$ and $\mathrm{d}+\mathrm{p}$ interactions at $\mathrm{E}_{k i n}=1.25 \mathrm{GeV}$ [12], i.e. just below the free eta meson production threshold, gave a chance to understand the important contributions to the di-electron spectra in $\mathrm{N}+\mathrm{N}$ collisions at these low bombarding energies.

Figure 1 shows the dielectron invariant mass spectrum obtained in $p+p$ and in quasi free $n+p$ reactions. From the direct comparison of the measured spectra one can notice a strong difference when going from $p+p$ to $n+p$ results. An especially striking difference at the high invariant mass is visible. The model description implemented in PLUTO assumed that the essential contribution at this energy comes from $\pi^{0}, \Delta(1232)$ and $\eta$ Dalitz decays and is shown in the figure 1 . The uncertainty in the contribution from the $\Delta(1232)$ due to the not well known electromagnetic transition form-factor of the decay of $\Delta \rightarrow N e^{+} e^{-}$is marked by shaded area in this figure. Although the the $\mathrm{p}+\mathrm{p}$ case can be described by this approach it is clearly visible that the yield in quasi-free $n+p$ collision is significantly underestimated. Due to the neutron momentum distribution inside the deuteron, the eta meson could be produced in this reaction and contributed to the dielectron spectra. This contribution is well known[12] and appeared not to be sufficient to explain the data. 

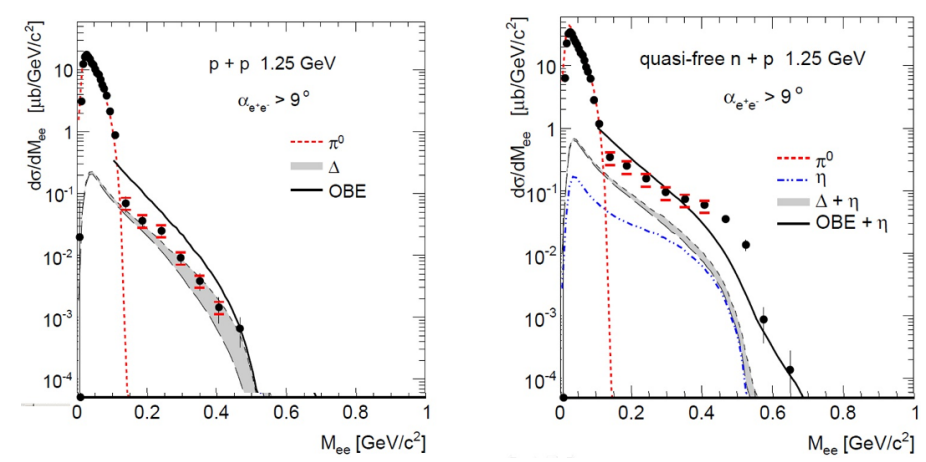

Figure 1. Differential cross section of $e^{+} e^{-}$pairs as a function of invariant mass measured in $\mathrm{p}+\mathrm{p}$ (left) and in quasi-free $\mathrm{n}+\mathrm{p}$ (right) reactions at $1.25 \mathrm{GeV}$ [12]. Individual contributions from $\pi^{0}, \Delta(1232)$ and $\eta$ Dalitz and prediction from the One Boson Exchange Model are compared to the data.

Another approach towards understanding the data is to compare them to the One Boson Exchange (OBE) model calculations. In this approach the parametrization of the calculated differential cross sections from [13] has been included. In addition, an isotropic virtual photon emission and corrections due to the final state interactions of $\mathrm{N}+\mathrm{N}$ have been implemented. As a result a significant overestimation of the $p+p$ data and disagreement with $n+p$ data have been obtained (figure 1). Although the
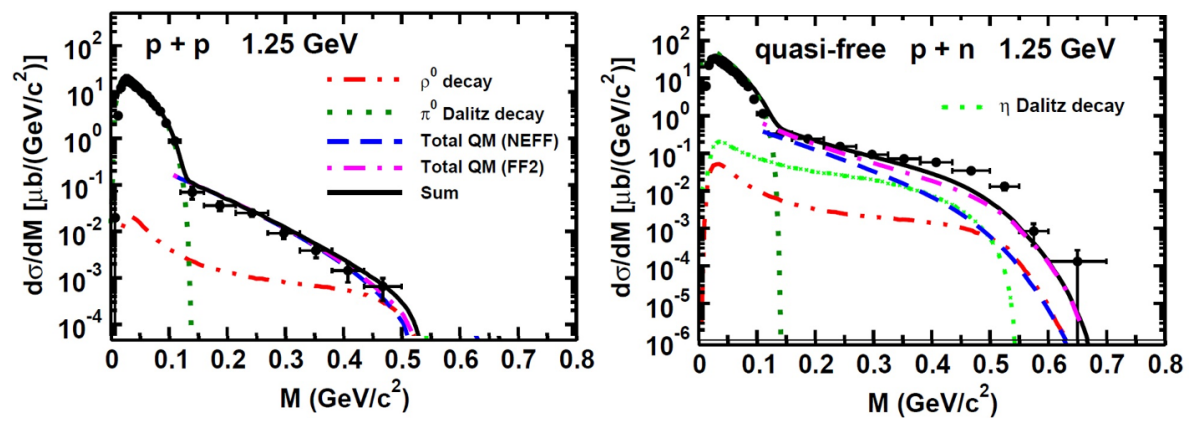

Figure 2. Differential cross section of $e^{+} e^{-}$pairs as a function of invariant mass measured in $\mathrm{p}+\mathrm{p}$ (left) and in quasi-free $\mathrm{n}+\mathrm{p}$ (right) reactions at $1.25 \mathrm{GeV}$ [12] compared to the OBE model predictions [14] w/o(marked as NEFF) and with(marked as FF2) electromagnetic form factor of charged pions.

initial attempts based on the one-boson exchange model (OBE) to describe these results did not fully succeed, more recent OEB calculations [14] shown in figure 2 come closer in describing the HADES $p+p$ and $n+p$ data. In this particular approach the electromagnetic form factor of the charged pion is taken into account. It plays a significant role in case of the $n+p$ reaction due to the possibility of charged pion exchange.

It is also interesting to note that recent model predictions based on GiBUU [15] and HSD [16] underestimate the $n+p$ data and the understanding of processes contributing to the dielectron production in elementary collisions is not settled. 
The low statistic and low resolution DSL results showing the ratio of dielectron yields of $p+d$ to $p+p$ [17] are summarized in Figure 3. As seen in this plot the isospin plays an important role in the energy region below $3 \mathrm{GeV}$ and reaches maximum at $1.25 \mathrm{GeV}$. Any interpretation of results from heavy ion induced reactions at SIS18 energies and at low SIS100 energies will strongly depend on the understanding of the isospin asymmetry described above. To gain further insight, the HADES

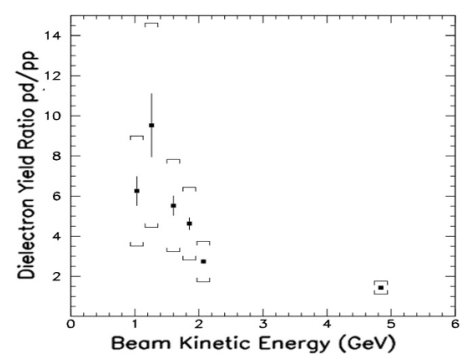

Figure 3. The ratio of the dielectron yields in the $p+d$ and $p+p$ systems [17] presented as a function of beam energy. Only pairs with masses greater than $0.15 \mathrm{GeV} / \mathrm{c}^{2}$ are included.

group performed measurements of inclusive di-electron production in $\mathrm{C}+\mathrm{C}$ at $2 \mathrm{~A} \mathrm{GeV} \mathrm{[18]} \mathrm{and}$ in $\mathrm{Ar}+\mathrm{KCl}$ at $1.76 \mathrm{~A} \mathrm{GeV} \mathrm{[19].} \mathrm{For} \mathrm{both} \mathrm{reaction} \mathrm{systems} \mathrm{a} \mathrm{significant} \mathrm{excess} \mathrm{of} \mathrm{di-electron} \mathrm{yield}$ above the known contributions has been observed.

Figure 4-left presents the reconstructed $e^{+} e^{-}$mass measured in $\mathrm{Ar}+\mathrm{KCl}$ collisions at $1.76 \mathrm{~A} \mathrm{GeV}$. The data are compared to a thermal dielectron cocktail of mesonic sources $\left(\pi^{0}, \eta, \omega\right)$ after freeze-out [19]. A strong excess yield over the freeze-out components has been found and is marked in figure 4-left. It has been demonstrated that light systems, like $\mathrm{C}+\mathrm{C}$, can be described in first order as an inco-
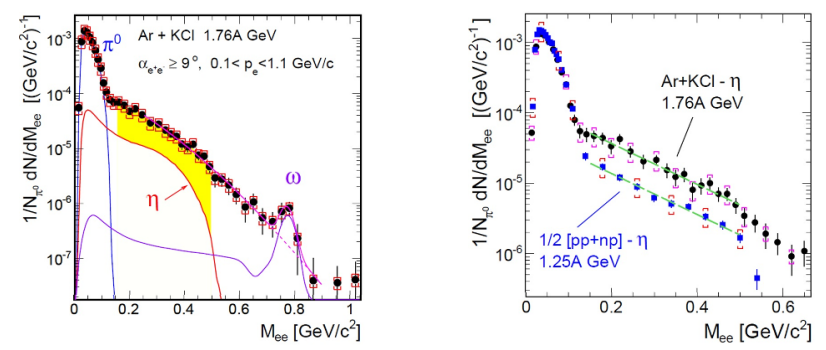

Figure 4. (Left) Reconstructed $e^{+} e^{-}$mass distribution in $\mathrm{Ar}+\mathrm{KCl}$ collisions at $1.76 \mathrm{AGeV}$ compared to freezeout sources of the dielectron cocktail $\left(\pi^{0}, \eta, \omega\right)$ [19]. (Right) Comparison of the $\eta$-subtracted $\mathrm{Ar}+\mathrm{KCl} e^{+} e^{-}$invariant mass distribution with an isospin-averaged reference from $p+p$ and $n+p$ data [12].

herent superposition of elementary $\mathrm{N}+\mathrm{N}$ processes [12]. The isospin-averaged spectra measured at $1.25 \mathrm{GeV}$ can serve as $\mathrm{N}+\mathrm{N}$ reference for the $\eta$-subtracted pair yield reconstructed in $\mathrm{Ar}+\mathrm{KCl}$ collisions at $1.76 \mathrm{~A} \mathrm{GeV}$ as shown in figure 4-right. The $\eta$-subtracted $\mathrm{Ar}+\mathrm{KCl}$ spectrum differs by a factor of $2.5-3$ from the $\mathrm{N}+\mathrm{N}$ reference for invariant masses between 0.15 and $0.5 \mathrm{GeV} / \mathrm{c}^{2}$. Based on this result one sees that heavier colliding systems can not be described anymore as a superposition of independent $\mathrm{N}+\mathrm{N}$ collisions and complex models involving multi-body and multistep processes and in-medium modifications are needed. 
It is of great importance to extend the investigation further above the $\mathrm{Ar}+\mathrm{KCl}$ colliding system and towards higher energies. Recently, the HADES Collaboration has collected data for $\mathrm{Au}+\mathrm{Au}$ at $1.25 \mathrm{~A} \mathrm{GeV}$ which is the highest energy available at SIS18 for this colliding system.

\subsection{High-mass pairs from omega decays}

The high-mass di-electron pairs are of particular importance as it is expected that the study of the omega signal provides insight into in-medium vector meson modifications. The di-electron data in the $\mathrm{Ar}+\mathrm{KCl}$ run show a pronounced $\omega$-meson signal (see figure 4-left ) which was observed for the first time at a beam energy well below the production threshold in free $\mathrm{N}+\mathrm{N}$ collisions $\left(\mathrm{E}_{t h r}^{N N}=1.89 \mathrm{~A} \mathrm{GeV}\right)$. The reconstructed signal in the $\omega$-pole mass region contains about 40 pairs, which does not allow any analysis of in-medium modifications.

In a subsequent experiment campaign, the HADES group has measured with high resolution and good event statistics inclusive di-electron invariant mass distributions in proton induced reactions on hydrogen and nuclear targets. The data for the elementary $\mathrm{p}+\mathrm{p}$ collisions at $\mathrm{E}=3.5 \mathrm{GeV}$ kinetic beam energy [20] are compared to theoretical predictions and allow to construct a reference spectrum for the extraction and possible interpretation of effects in the $\mathrm{p}+{ }^{93} \mathrm{Nb}$ system [21], in particular in terms of vector meson production. As shown in figure 5, a clear $\omega$-meson signal is seen in the $\mathrm{p}+{ }^{93} \mathrm{Nb}$ reaction. As figure 5 shows a strong modification of the shape of the spectrum for $e^{+} e^{-}$momenta
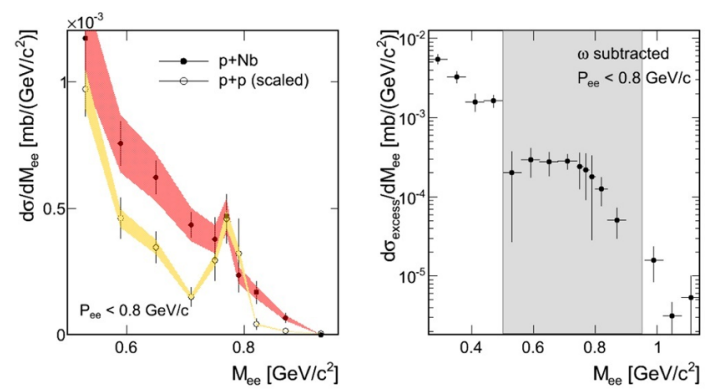

Figure 5. (Left) Comparison of the invariant mass spectra for $e^{+} e^{-}$pairs with $\mathrm{P}_{e e}<0.8 \mathrm{GeV} / \mathrm{c}$. The $\mathrm{p}+\mathrm{p}$ data have been scaled according to a Glauber model, details in [21]. Systematic uncertainties are also shown. (Right) Excess yield in $\mathrm{p}+\mathrm{Nb}$ data after subtraction of the $\mathrm{p}+\mathrm{p}$ reference data and after $\omega$ subtraction.

$P_{e e}<0.8 \mathrm{GeV} / \mathrm{c}$ has been observed. This can be interpreted as a decrease of the $\omega$ yield with an additional $\rho$-like contribution as suggested in [21].

\section{Hadron production measured with HADES}

Although HADES was designed primarily for di-electron measurements, it has also shown an excellent capability for the identification of charged hadrons like $\mathrm{p}, \pi^{+}, \pi^{-}$and $K^{+}, K^{-}$. Hence, their production together with those of $\mathrm{K}^{0} \rightarrow \pi^{+} \pi^{-}, \Lambda \rightarrow \mathrm{p} \pi^{-}, \phi \rightarrow K^{+} K^{-}$and even $\Xi \rightarrow \Lambda \pi^{-}$has been studied in detail for the $\mathrm{Ar}+\mathrm{KCl}$ system [22-24] at $\mathrm{E}=1.756 \mathrm{~A} \mathrm{GeV}$. A combined and inclusive identification of $K^{+} K^{-}$pairs and $\phi$ mesons was performed for the first time in the same experiment at a subthreshold beam energy for $K^{-}$and $\phi$ production. The obtained $\phi / K^{-}$ratio of $0.37 \pm 0.13 \%$ indicates that $18 \pm 7 \%$ of the kaons stem from $\phi$ decays. This significant contribution to the $K^{-}$production has to be included in the models. 

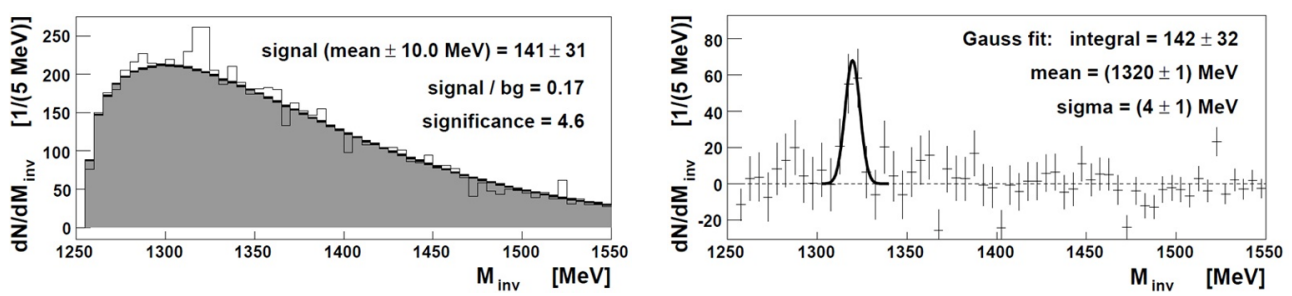

Figure 6. (Left) $\Lambda-\pi^{-}$invariant mass distribution. Gray histogram shows combinatorial background produced via event mixing. (Right)The invariant mass distribution after background subtraction with a Gaussian fit to the $\Xi^{-}$signal.

Taking advantage of the excellent vertexing capability of the HADES spectrometer and high statistic data it was possible to investigate the deep sub-threshold production of the double strange hyperon $\Xi^{-}$in $\mathrm{Ar}+\mathrm{KCl}$ at $1.76 \mathrm{~A} \mathrm{GeV} \mathrm{[25].} \mathrm{The} \Xi^{-}$signal reconstructed with a significance of about five is shown in figure 6. It should be mentioned that this signal has been reconstructed far below the free $\mathrm{N}+\mathrm{N}$ threshold, i.e. at $\sqrt{s_{N N}}-\sqrt{s_{t h r}}=-640 \mathrm{MeV}$ and is by about a factor of 10 stronger than any model predictions [25].

\section{HADES at FAIR}

With beam energies available from the present SIS18 (1-2 A GeV) accelerator one can investigate nuclear matter at inverse slope parameters (temperatures) of $\mathrm{T}=40-90 \mathrm{MeV}$ and baryon densities of 1-3 $\rho_{0}$. The beam energies of the planned SIS100 accelerator in future FAIR facility will, however, allow to reach nuclear matter densities exceeding substantially those achievable at SIS18. Exploring this region of the phase diagram is the main purpose of the proposed future HADES programme at FAIR. So far, there are no data on dilepton production available for the energy regime between 2 and $40 \mathrm{~A} \mathrm{GeV}$. Hence, this regime is "terra incognita" for di-electron measurements. HADES has recently demonstrated excellent performance by measuring $\mathrm{Au}+\mathrm{Au}$ at $1.25 \mathrm{~A} \mathrm{GeV}$ and has proven its readiness for future SIS100 experimental campaigns.

\section{Summary}

The HADES experiment has provided systematic results on $e^{+} e^{-}$production in elementary and heavy ion collisions. The analysis of $\mathrm{C}+\mathrm{C}$ collisions at $1 \mathrm{~A} \mathrm{GeV}$ delivered high quality data and confirmed the old DLS findings, indicating significant lack of knowledge on the dilepton production processes at these low bombarding energies. The set of heavy ion experiments supplemented by proton induced reactions delivered a better understanding of the origin of the measured pair excess and point to the importance of low-energy processes like pion production and bremsstrahlung. Differences in model descriptions of the measured di-electron spectra in $p+p$ and $n+p$ collisions show lack of understanding the different contributions. The signal from direct $\omega$ - meson decays measured for the first time in the SIS18 energy range demonstrated the excellent performance of the HADES spectrometer in terms of mass resolution, particle identification and DAQ system.

The HADES results on strangeness production shed new light on the understanding of kaon production mechanisms in HI collisions, by providing e. g. compelling evidence that the contribution from 
the $\phi$ decay to the $K^{-}$yield has to be also taken into account. The measured hadron yields have in general been found in good agreement with statistical model predictions, besides the $\Xi^{-}$, which is produced far below its production threshold and shows a considerable deviation from the statistical model.

The recently conducted and still being analyzed measurement of Au+Au collisions at SIS18 as well as future experiments planned at the FAIR facility will deliver new valuable data on di-electron and strangeness production.

\section{Acknowledgments}

The HADES Collaboration gratefully acknowledges the support by BMBF grants 06DR9059D, 05P12CRGHE, 06FY171, 06MT238 T5, and 06MT9156 TP5, by HGF VH-NG-330, by DFG EClust 153, by GSI TMKRUE, by the Hessian LOEWE initiative through HIC for FAIR (Germany), by EMMI GSI, by grant GA CR 13-067595 (Czech Rep.), by grant NN202198639 (Poland), by grant UCY-10.3.11.12 (Cyprus), by CNRS/IN2P3 (France), by INFN (Italy), and by EU contracts RII3-CT2005-515876 and HP2 227431.

\section{References}

[1] C. Sturm et al. (KaoS Collab.), Phys. Rev. Lett. 86, 39 (2001).

[2] J. Schaffner-Bielich et al. Nucl. Phys. A 625, 325 (1997); W. Cassing et al., Nucl . Phys. A 614 415 (1997).

[3] G. Agakishiev et al. Eur.Phys.J.A41:243-277,2009

[4] R.J. Porter et al., (DLS Collab.), Phys. Rev. Lett. 79 (1997) 1229.

[5] G. Agakichiev et al., (CERES Collab.), Eur. Phys. J. C 41 (2005) 475

[6] R. Arnaldi et al., (NA60 Collab.),Phys. Rev. Lett. 96 (2006) 162302.

[7] H. van Hees et al., Phys. Rev. Lett. 97 (2006) 102301; T. Renk et al. Eur. Phys. J. C 49 (2007) 219

[8] C. Ernst et al., Phys. Rev. C 58 (1998) 447; W. Cassinget al., Phys. Rep. 308 (1999) 65; K. Shekhter et al., Phys. Rev. C 68 (2003) 014904.

[9] G. Agakichiev, et al., (HADES Collab.), Physics Letters B 663 (2008) 43-48.

[10] M I. Krivoruchenko, B V. Martemyanov, A. Faessler, and C. Fuchs, Ann. Phys. 296, 299 (2002).

[11] G. Agakishiev et al. (HADES Collab.) Phys.Rev. C85 (2012) 054005

[12] G. Agakishiev et al. (HADES Collab.), Phys. Lett. B 690, 118 (2010).

[13] L. P. Kaptari and B. Kämpfer, Nucl. Phys. A 764, 338 (2006), Phys. Rev. C 80, 064003 (2009).

[14] R. Shyam, U. Mosel, Phys. Rev. C 82, 062201 (2010).

[15] J. Weil, H. van Hees, and U. Mosel, Eur. Phys. J. A (2012) 48: 111

[16] E.L. Bratkovskaya et al. arXiv:1301.0786v2.

[17] W. Wilson et al., Phys. Rev. C 57 (1998).

[18] G. Agakichiev et al., (HADES Collab.), Phys. Rev. Lett. 98 (2007) 052302

[19] G. Agakishiev et al., (HADES Collab.) Phys.Rev. C84 (2011) 014902

[20] G. Agakishiev et al., (HADES Collab.) Eur.Phys.J. A48 (2012) 64

[21] G. Agakishiev et al., (HADES Collab.) Phys.Lett. B715 (2012) 304

[22] G. Agakishiev et al., (HADES Collab.) Phys.Rev.C82:044907,2010

[23] G. Agakishiev et al., (HADES Collab.) Phys.Rev.C80:025209,2009.

[24] G. Agakishiev et al., (HADES Collab.) Eur.Phys.J.A47:21,2011.

[25] G. Agakishiev et al. (HADES Collab.) Phys. Rev. Lett. 1003 (2009) 132301 